Proc. Indian Acad. Sci. (Earth Planet. Sci.), Vol. 105, No. 1, March 1996, pp. 63-80.

(C) Printed in India.

\title{
Understanding the turbulent structure of the atmospheric boundary layer: A diagnostic approach
}

\author{
M CHATTERJEE, G K SEN and D K SINHA \\ Centre for Atmospheric Sciences, University of Calcutta, 92, A.P.C. Road, Calcutta 700009, \\ India
}

\begin{abstract}
In this paper, we have attempted a diagnostic study of the turbulence characteristics of the ABL by means of two one-dimensional models. The first model uses a first order non-local closure, based on the Transilient Turbulence Theory, for parameterizing turbulent fluxes. while the second model uses second order local closure for parameterizing these. The models have been applied to conduct case studies using the Kytoon data taken at Kharagpur, during 17 th -21 st June, 1990, as part of the MONTBLEX programme.

Our findings bring out various interesting features regarding the non-local and local turbulent statistics such as kinematic fluxes, turbulence kinetic energy, vertical velocity variance, the contribution of the eddies of various sizes to the fluxes at different levels and the mixing lengths. The one-dimensional anisotropy of the turbulent eddies has been revealed by the findings from the transilient model. The vertical variation of the turbulence kinetic energy, as computed directly by the second order model, is found to be strongly correlated with the vertical velocity variance. In particular, for stably stratified boundary layers, identification of two distinct zones of the turbulence kinetic energy and corresponding vertical velocity maxima is possible, which has been interpreted as positive evidence of patchy turbulence in the boundary layer.
\end{abstract}

Keywords. Atmospheric boundary layer; non-local closure; local closure; turbulence structure.

\section{Introduction}

The most important and interesting feature within the atmospheric boundary layer $(\mathrm{ABL})$ is the turbulent eddies that are generated due to external forcings such as solar heating of the earth's surface and the frictional drag it exerts on the layers of air above it, radiative cooling, wind shear, etc. Depending on the existing mean flow conditions within the $A B L$, turbulence increases or decays with time and the eddies interact with the surrounding air and with each other, thereby transporting and exchanging mass, momentum, heat, moisture and energy at various levels.

The wide range or spectrum of the turbulent eddy sizes is difficult to take into account completely in observational and theoretical investigations attempting in-depth studies of the various $\mathrm{ABL}$ processes. Simplifying assumptions, such as concentrating on a particular range of eddy sizes and employing statistical methods for quantifying turbulence, have been adopted. These lead us to a set of equations governing ABL flow which, in addition to the mean flow terms, now contain non-linear turbulence terms. These non-linear terms are the statistical moments of various orders involving the turbulent components of wind, temperature and moisture, and mathematically represent processes such as turbulent fluxes, turbulence kinetic energy (TKE) etc. As first recognised by Keller and Friedman (1924), the number of equations governing 
ABL flow is less than the number of unknowns contained in them. This has resulted in the well-known closure problem in which the unknown turbulent quantities have to be specified in terms of known parameters in order to obtain solutions of these equations.

Two principal classes of closure methods are usually used in order to solve the ABL equations (Stull 1988). Local closure methods are based on analogy with the process of molecular diffusion and assume that turbulent transport is a diffusive process. The unknown turbulent quantities at any point in space are parameterized in terms of the known quantities such as the observed wind, temperature, humidity or their gradients at that point. The well-known bulk methods and $\mathrm{K}$-theory methods are examples of local closure parameterization. Local closure methods have been used extensively over a long period of time by investigators, and references become too numerous to cite. Thus it may be appropriate to refer to reviews by Bhumralkar (1976), McBean et al (1979), Nieuwstadt and van Dop (1984), Stull (1988) and others.

The non-local closure methods of parameterization are based on the idea that turbulent processes are advective rather than diffusive and the unknown quantities at any point in space are expressed in terms of known quantities at various points in space. Large eddy simulation techniques (Deardorff (1972), Wyngaard and Brost (1984)) and the transilient turbulence theory (Stull 1984, 1988, 1992), hereafter referred to as Stull $(a, b, c)$, are examples of non-local closure methods.

However, none of these closure methods, or the parameterization schemes adopted in them, are exact. Both have advantages and disadvantages and their applications are dictated by the particular physical processes being investigated. It has been observed (Stull 1988) that local closure methods of second and higher orders, and the non-local closure methods in general, provide more accurate and realistic descriptions of turbulence within the ABL.

In the present paper, we have attempted to investigate turbulence within the ABL through two numerical models. Both are diagnostic, one-dimensional models. For parameterizing turbulent fluxes, one of them uses a first order, non-local closure method based on transilient turbulence theory, and the other model uses second order local closure method for doing the same. Using the available Kytoon data for Kharagpur, taken as part of the MONTBLEX programme, we have applied these two models to study the structure of the ABL during 17th - 21st June, 1990.

The findings from these models reveal various interesting aspects of the turbulent transports in the ABL, such as non-local and local kinematic fluxes of heat and momentum, the contribution of eddies of different sizes to the non-local fluxes at each level, the mixing length profiles ard the one-dimensional anisotropy of the turbulent eddies. An interesting feature revealed from the results of the second order model is the strong correlation between the TKE and the vertical velocity variance maxima. Interestingly, for stable boundary layers, we could distinguish clearly between two separate zones within the ABL where these peaks lie. These zones may be interpreted as zones of patchy turbulence, a commonly observed feature in the stable boundary layer.

\section{The non-local closure model: Transilient turbulence theory}

Detailed description of the transilient turbulence theory (TTT) is available in Stull $(a, b, c)$. Briefly stated, TTT is based on the fact that the larger turbulent eddies are organised, 
coherent structures, which transport turbulent properties such as momentum, heat, moisture, pollutants etc, across finite distances in space, in a manner analogous to the process of advection, before the smaller sized eddies begin to do so. TTT thus allows the incorporation of the effects of turbulent mixing not only between adjacent layers or grid boxes (as in the local closure methods), but also between grid points separated by finite distances, which is extremely advantageous.

Proceeding as in Stull $(a, b, c)$, we discretize the vertical column of air over the observing station (Kharagpur) into $N$ not necessarily equal-sized grid boxes of length $\Delta Z$. Grid indices are placed at the box centres with $i$ denoting a reference (destination) box and $j$ denoting other source boxes.

At any instant of time $t$ (seconds), all the grid boxes contain air which have some average property of state $\bar{\xi}_{i}(t)$, which may physically represent its mass, momentum, temperature, humidity etc. After a small interval of time $\Delta t$, turbulence, if present, will cause mixing of air between boxes $i$ and $j$. If $c_{i j}(t, \Delta t)$ denotes the fraction of air in box $i$ after it has mixed with air from box $j$, and assuming that during the time $\Delta t$ air from the box $j$ retains and carries its original state property $\xi_{j}(t)$ into box $i$, the average property of state $\bar{\xi}_{i}(t)$ will change to $\bar{\xi}_{i}(t+\Delta t)$ according to the relation:

$$
\bar{\xi}_{i}(t+\Delta t)=\sum_{j=1}^{N} c_{i j}(t, \Delta t) \bar{\xi}_{j}(t)
$$

The elements $c_{i j}(t, \Delta t)$ of the transilient matrix $C(t, \Delta t)$ are the unknown quantities which must be parameterized in terms of the known mean flow variables. To make the parameterization more realistic, the continuous response of the ABL flow to the external forcings and conditions at the earth's surface should be taken into account. Any external or dynamical forcing at the earth's surface such as insolation, frictional drag, wind shear, etc., causes destabilization of the mean flow in the ABL and generation of turbulence. Turbulent mixing then tends to restabilize the flow to a new equilibrium state. Mathematically this concept is written as (Stull (c)):

$$
\begin{aligned}
& \bar{\xi}_{i}^{*}(t+\Delta t)=\bar{\xi}_{i}(t)+\Delta t \cdot(\text { net source at } Z=i \Delta Z), \\
& \bar{\xi}_{i}(t+\Delta t)=\sum_{j=1}^{N} c_{i j}(t, \Delta t) \bar{\xi}_{j}^{*}(t+\Delta t),
\end{aligned}
$$

with $c_{i j}(t, \Delta t)=$ function $\left(u_{i}, u_{j}, v_{i}, v_{j}, \theta_{i}, \theta_{j}, \cdots\right)$.

In the present study, the responsive parameterization scheme used is known as the mixing potential approach and had been proposed by Stull and Driedonks (1987). The mixing potential $Y_{i j}$ for each source destination box is computed from the TKE budget equation which in the non-local framework has the form:

$$
Y_{i j}=\left[\frac{\Delta t \cdot T_{0}}{\left(\Delta_{i j} Z\right)^{2}}\left(\Delta_{i j} u\right)^{2}+\left(\Delta_{i j} v\right)^{2}-\frac{g\left(\Delta_{i j} Z\right)\left(\Delta_{i j} \theta\right)}{\bar{\theta} R_{c}}\right]-\frac{D_{y} \cdot \Delta t}{T_{0}} \forall i \neq j,
$$

where the notation $\Delta_{i j} X=X_{i}-X_{j}$ implies the non-local difference between $X_{i}$ and $X_{j}$. In (2.3), the last term represents the TKE dissipation. Also, the parameters $T_{0}(=1000 \mathrm{~s})$ and $R_{c}(=0.25)$ represent the time scale and the critical Richardson number respectively. Observed values of the wind and temperature are used to compute the $Y_{i j}$. 
The elements $c_{i j}(t, \Delta t)$ are then computed from:

$$
\begin{aligned}
& c_{i j}=\frac{Y_{i j}}{\|Y\|}, \quad i \neq j, \\
& c_{i i}=1-\sum_{\substack{j=1 \\
j \neq i}}^{N} c_{i j},
\end{aligned}
$$

where $\|Y\|$ is the maximum row norm of the matrix $Y_{i j}$.

The non-local flux $F_{k}(t, \Delta t)$ across the level $k$ (that is at height $Z=k \Delta Z$ ) is computed from (Stull and Driedonks 1987):

$$
F_{k}(t, \Delta t)=F_{k-1}(t, \Delta t)+\frac{\Delta Z}{\Delta t} \cdot \sum_{j=1}^{N} c_{k j}(t, \Delta t)\left[\bar{\xi}_{k}(t)-\bar{\xi}_{j}(t)\right],
$$

with $F_{k}(t, \Delta t)=0$ at $k=0$ (that is across the earth's surface) and also at $k=N$ (at the model top).

The transport spectral components $F_{k / m}(t, \Delta t)$ give the contribution of the eddies of size $m \Delta Z$ to the total flux across the level $k$ (that is at $Z=k \Delta Z$ ). These may be computed from Stull $(b, c)$ :

$$
F_{k / m}(t, \Delta t)=\frac{\Delta Z}{\Delta t} \cdot \sum_{i=1}^{k} \sum_{j=k+1}^{N} \delta_{m, \mid i-j}\left[c_{i j}(t, \Delta t) \bar{\xi}_{i}(t)-c_{j i}(t, \Delta t) \bar{\xi} Z_{j}(t)\right],
$$

where $\dot{\delta}_{m, n}$ denotes the Kronecker delta function.

The mixing length at any level $k(Z=k \Delta Z)$ is given by:

$$
l_{k}=0.5 \Delta Z \cdot \sum_{i=1}^{N}\left[c_{i k}(t, \Delta t)+c_{k i}(t, \Delta t) \cdot|i-k| .\right.
$$

To get some idea about the one-dimensional anisotropy of turbulence in the ABL, the mixing length $l_{k}$ is separated into upward and downward transport from the source box $j$ :

$$
\begin{array}{r}
l_{j} \uparrow(t, \Delta t)=\frac{\Delta Z \cdot \sum_{i=j}^{N} c_{i j}(t, \Delta t) \cdot|i-j|}{\sum_{i=j}^{N} c_{i j}(t, \Delta t)}, \\
l_{j \downarrow} \downarrow(t, \Delta t)=\frac{\Delta Z \cdot \sum_{i=1}^{j} c_{i j}(t, \Delta t) \cdot|i-j|}{\sum_{i=1}^{j} c_{i j}(t, \Delta t)},
\end{array}
$$

and an upward and downward transport to the destination box $i$ :

$$
l_{i} \uparrow(t, \Delta t)=\frac{\Delta Z \cdot \sum_{j=1}^{i} c_{i j}(t, \Delta t) \cdot|i-j|}{\sum_{j=1}^{i} c_{i j}(t, \Delta t)},
$$




$$
l_{i} \downarrow(t, \Delta t)=\frac{\Delta Z \cdot \sum_{j=i}^{N} c_{i j}(t, \Delta t) \cdot|i-j|}{\sum_{j=i}^{N} c_{i j}(t, \Delta t)}
$$

The transilient model was run with a vertical grid resolution of $\Delta Z=10 \mathrm{~m}$ and a time interval of $\Delta t=600 \mathrm{~s}$. Model results have been discussed in $\S 4$.

\section{The second order local closure model}

The second order local closure model used in this study is a simplified version of the model given by Donaldson (1973). The simplifying assumptions we have adopted are briefly described below.

Being primarily concerned with a diagnostic study, the steady state version of the prognostic equations of turbulence variances have been used. The triple correlation term $\overline{w^{\prime} \theta^{\prime 2}}$ has been omitted following arguments by Hogstorm (1990) that suggest that this term is usually negligible near the surface and throughout the ABL. Furthermore, in the case studies attempted here, the ABL usually had a very weak or nearly neutral stratification and turbulence was weak. We have thus neglected the triple correlation terms $\overline{u^{\prime} w^{\prime 2}}$ and $\overline{v^{\prime} w^{\prime 2}}$ in the equations for $\overline{u^{\prime 2}}$ and $\overline{v^{\prime 2}}$. Terms such as $\overline{u^{\prime} \theta^{\prime}}, \overline{v^{\prime} \theta^{\prime}}$, and $\overline{u^{\prime} v^{\prime}}$ occurring in these equations have also been neglected. These simplifications enable us to obtain a set of coupled, first order ordinary differential equations:

$$
\begin{aligned}
& \frac{\partial u}{\partial t}=-f\left(v-v_{g}\right)+\frac{\partial}{\partial Z}\left(\overline{w^{\prime} u^{\prime}}\right), \\
& \frac{\partial v}{\partial t}=f\left(u-u_{g}\right)+\frac{\partial}{\partial Z}\left(\overline{w^{\prime} v^{\prime}}\right), \\
& \frac{\partial \theta}{\partial t}=\frac{\partial}{\partial Z}\left(\overline{w^{\prime} \theta^{\prime}}\right) \\
& { }^{\prime} u^{\prime} \frac{\partial u}{\partial Z}+\overline{w^{\prime} v^{\prime}} \frac{\partial v}{\partial Z}-\frac{g}{\theta} \overline{w^{\prime} \theta^{\prime}}+\frac{\partial}{\partial Z}\left(\Lambda_{e} e^{1 / 2} \frac{\partial e}{\partial Z}\right)+\frac{e^{3 / 2}}{\Lambda_{1}}=0, \\
& \overline{w^{\prime 2}} \frac{\partial u}{\partial Z}+\frac{e^{1 / 2}}{\Lambda_{2}} \overline{w^{\prime} u^{\prime}}=0, \\
& \overline{w^{\prime 2}} \frac{\partial v}{\partial Z}+\frac{e^{1 / 2}}{\Lambda_{2}} \overline{w^{\prime} v^{\prime}}=0 \\
& \frac{2 g}{\theta} \overline{w^{\prime} \theta^{\prime}}+\frac{e^{1 / 2}}{\Lambda_{3}}\left(\overline{w^{\prime 2}}-\frac{2}{3} e\right)=0, \\
& \overline{w^{\prime 2}} \frac{\partial \theta}{\partial Z}-\frac{g}{\theta} \overline{\theta^{\prime 2}}+\frac{e^{1 / 2}}{\Lambda_{3}} \overline{w^{\prime} \theta^{\prime}}=0 \text {, } \\
& \overline{2 w^{\prime} \theta^{\prime}} \frac{\partial \theta}{\delta Z}+\frac{e^{1 / 2}}{\Lambda_{4}} \overline{\theta^{\prime 2}}=0 \text {, }
\end{aligned}
$$


with

$$
\begin{aligned}
& \Lambda_{1}=5 \cdot 75 \Lambda, \Lambda_{2}=2 \cdot 08 \Lambda, \Lambda_{3}=2 \cdot 14 \Lambda \\
& \Lambda_{4}=7 \cdot 44 \Lambda \text { and } \Lambda_{e}=0 \cdot 2 \Lambda
\end{aligned}
$$

(Donaldson (1973)).

The system of equations (3.1) - (3.9) has been solved numerically, at first keeping the turbulence length scale $\Lambda$ constant and then allowing it to vary with height. The vertical grid chosen for both cases is identical to the one used for the transilient model.

For the second case, the simplest method for estimating the variation of $\Lambda$ with height is based on the comparison of the grid point values of the turbulent fluxes, $\overline{w^{\prime} \theta^{\prime}}, \overline{w^{\prime} u^{\prime}}, \overline{w^{\prime} v^{\prime}}$, obtained by numerically solving the diagnostic equations $(3.4)-(3.9)$ with those obtained from the transilient model. In this case, it is to be expected that a single grid point value of $\Lambda$ will not reproduce all the three fluxes simultaneously. In order to assess the profiles of $\Lambda$ for the case (A) where the effect of surface frictional drag is large and case (B) where the surface heat flux is dominant, two numerical experiments were performed. In experiment (A), a surface heat flux $\overline{w^{\prime} \theta^{\prime}}=-0.004 \mathrm{~K} \mathrm{~m} / \mathrm{s}$ and a drag coefficient $C_{D}=0.0039$ was provided. These inputs are the same as the ones used for the case study for 20 th June with the transilient model. In experiment $(B)$, the prescribed surface heat flux is $\overline{w^{\prime} \theta^{\prime}}=-0.006 \mathrm{~K} \mathrm{~m} / \mathrm{s}$ with a drag coefficient $C_{D}=0.0015$. The results have been discussed in $\S 4$.

\section{Case studies}

The non-local closure model described in $\S 2$ has been used to compute the vertical profiles of various ABL parameters over Kharagpur $(22.30 \mathrm{~N}, 87.2 \mathrm{E})$ during 19 th 21 st June, 1990 . The second order local closure model described in $\S 3$ has been used to obtain estimates of the TKE and turbulent vertical velocity variance at the same location for the period 17th - 21st June, 1990.

The data used are Kytoon observations of pressure, pressure height, dry and wet bulb temperatures, wind speed and direction taken at Kharagpur during the MONTBLEX programme. The time of observation for 17th - 20th June was 00 GMT $(0530$ local time) and 1130 GMT (1700 local time) for 21st June. These different timings enable us to study the early morning ABL (17th - 20th June) which is a continuation of the nocturnal stable boundary layer, and also the late afternoon ABL (21st June, 1990) as it changes from the daytime convective boundary layer to the nocturnal one.

The basic inputs to the transilient model are the observed potential temperature $\theta\left({ }^{\circ} \mathrm{K}\right)$ and the zonal and meridional wind components $u$ and $v(\mathrm{~m} / \mathrm{s})$ at $10 \mathrm{~m}$ intervals. These initial profiles of $\theta, u, v$, for 19th -21 st June have been shown in figure 1 . In figure 2(a), the vertical variation of the Richardson numbers computed from the observations for these three days have been shown. It is seen that on 19th June, the lowest $15 \mathrm{~m}$ of the $\mathrm{ABL}$ is unstable above which lies a stable layer, with highly stable zones around 70-80 m, 100-120 $\mathrm{m}$ and $155-211 \mathrm{~m}$ and the ABL top around $200 \mathrm{~m}$. On 20 th and 21 st June, the ABL stratification was weaker, with the lowest $15 \mathrm{~m}$ having a weakly stable or near neutral stratification on 20 th June and a weakly unstable or near neutral stratification on 21 st June. On 20 th June, stable stratification existed above this level, with the height of the ABL top around $175 \mathrm{~m}$, while on 21 st June the 

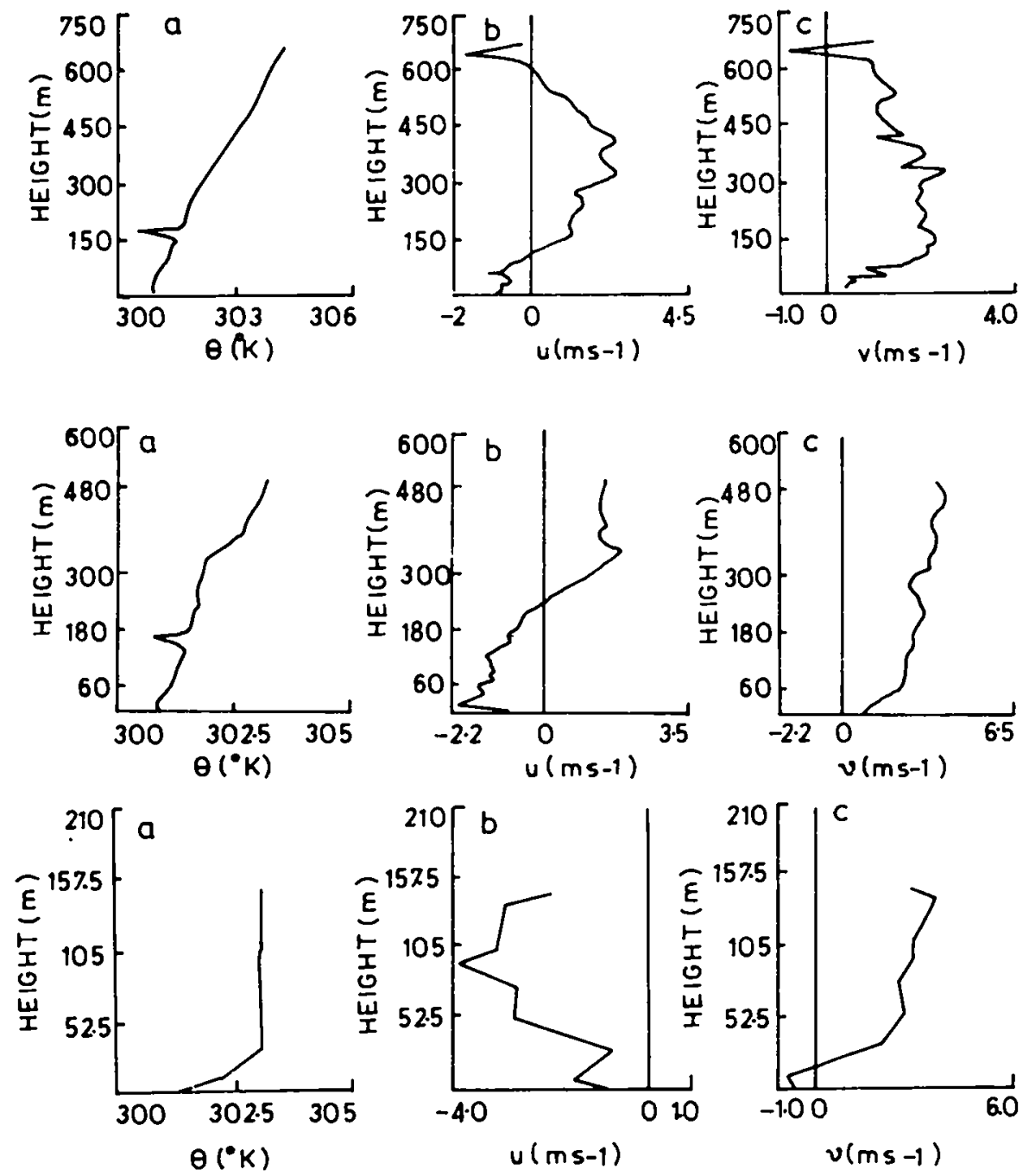

Figure 1. Observed profiles of: a) $\theta$, b) $u$ and c) $v$ for Kharagpur.

Top panel : 19th June, 1990, 00 GMT.

Middle panel : 20th June, $1990,00 \mathrm{GMT}$.

Bottom panel: 21 st June, 1990, $1130 \mathrm{GMT}$.

layers between $40-90 \mathrm{~m}$ were neutrally stratified, with the $A B L$ top around $125 \mathrm{~m}$. In this connection, we mention that discussions on local vis-a-vis non-local stability can be found in Stull (1991) and Stull (c).

In addition to these basic inputs, prescribed heat and momentum fluxes were provided at the lowest model level. These were estimated in the usual manner from the first two levels of observations, namely $3 \mathrm{~m}$ and $15 \mathrm{~m}$. After 10 minutes of transilient mixing, the model outputs consisting of vertical profiles of $\theta, u, v$ and fluxes $\overline{w^{\prime} \theta^{\prime}}, \overline{w^{\prime} u^{\prime}}, \overline{w^{\prime} v^{\prime}}$ have been displayed in figures 3,4 and 5 .

The Richardson number variation with height as computed from the model outputs has been shown in figure 2 (b). This may be compared with figure 2 (a) to get an idea 

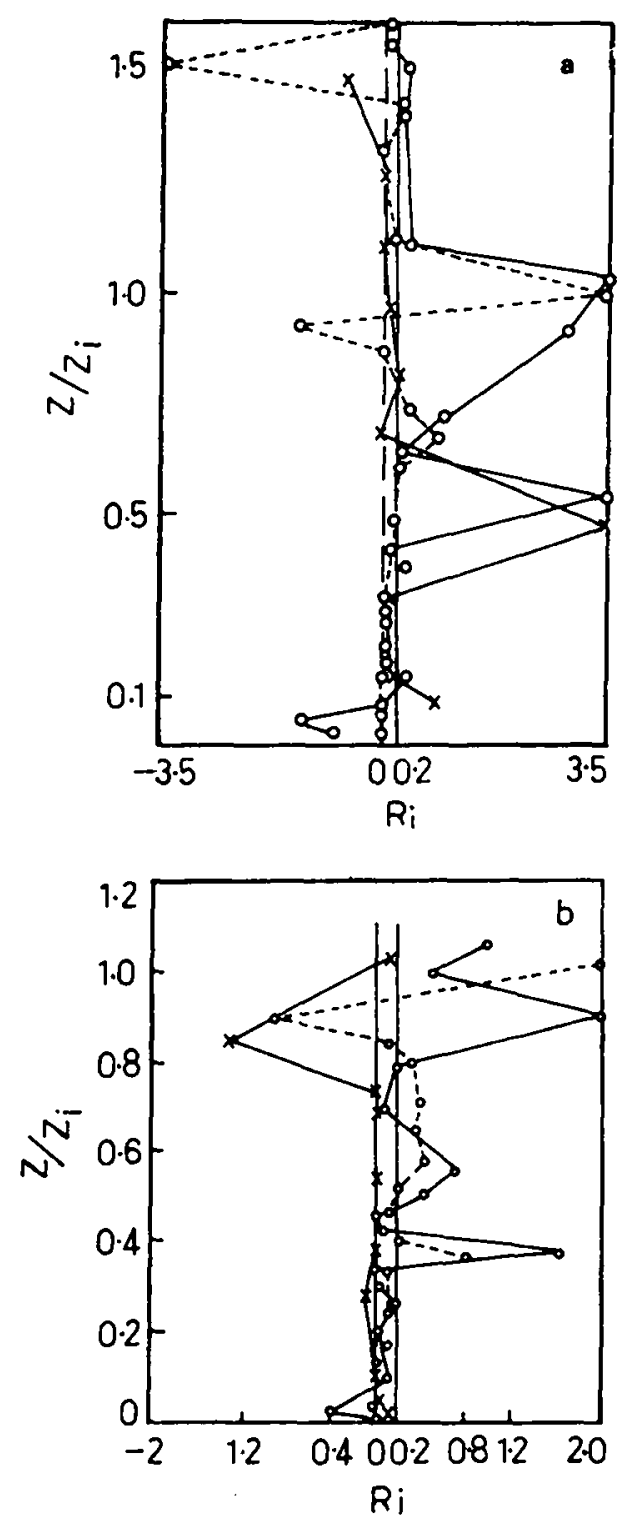

Figure 2. Richardson numbers computed from: a) Observed proliles and b) transilient model outputs. $O-.-019$ th June; $O-0^{--}-020$ th June and $x \quad x-\times 21$ st June.

about how the turbulent zones have changed within these 10 minutes. The heat flux. as seen from figures $3(\mathrm{~d}), 4(\mathrm{~d})$ and $5(\mathrm{~d})$ is negative up to a considerable height for all three days. This implies subsidence and downward transport of heat which is not uncommon in stably stratified zones. The ABL tops on these days have been estimated to be around $Z_{i}=230 \mathrm{~m}, 178 \mathrm{~m}$ and $85.5 \mathrm{~m}$ respectively. Thus for the early morning case studics, 19 th - 20th June, the ABL has warmed up as observed also from figures 3(a) and 4(a), and the tops have risen. As seen from figure 5(a), on 21 st June, although the temperature 

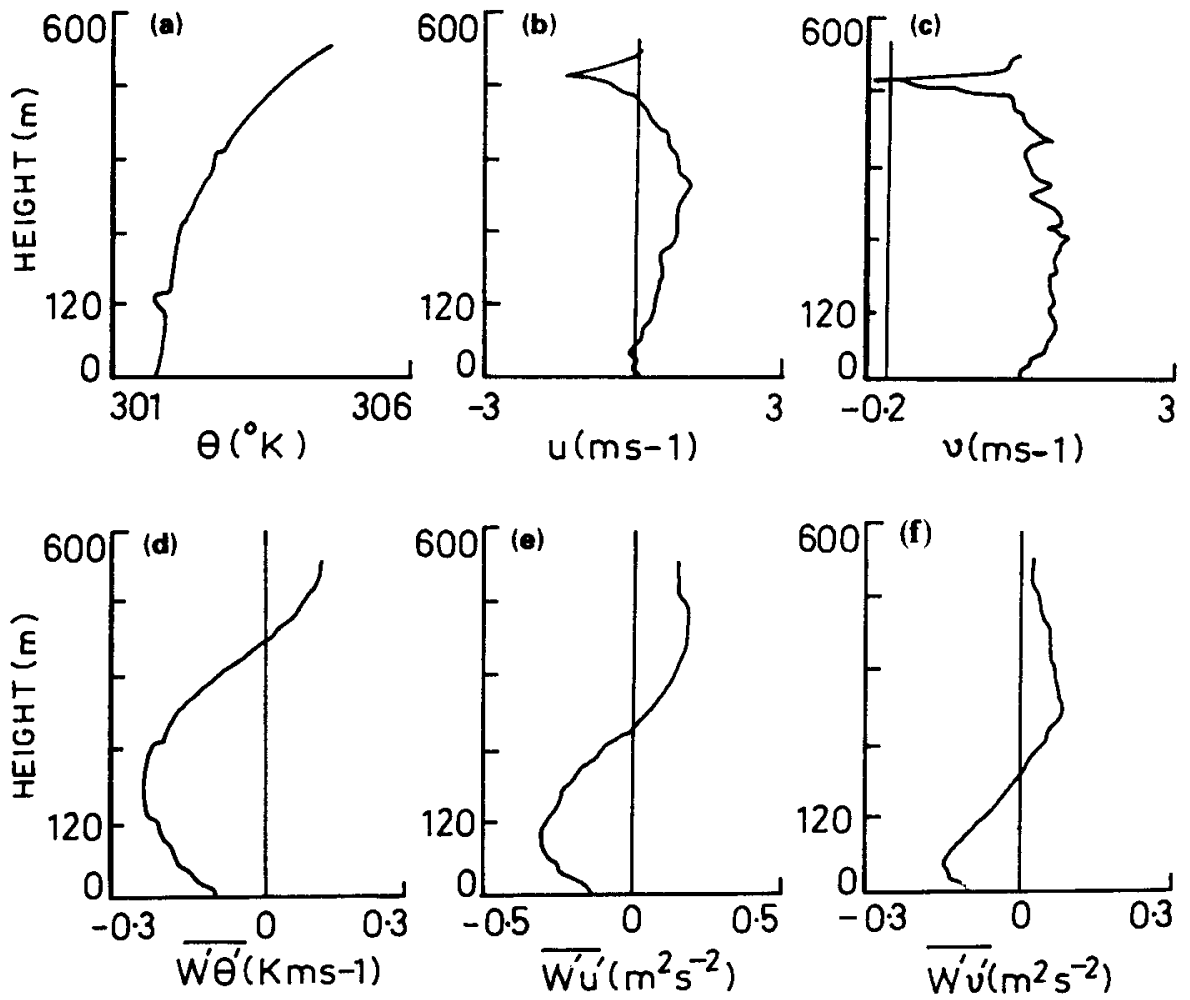

Figure 3. Transilient model outputs for 19th June, $1990-\theta, u, v, \overline{w^{\prime} \theta^{\prime}}, \overline{w^{\prime} u^{\prime}}$ and $\overline{w^{\prime} v^{\prime}}$.

has risen at the lowest level, temperature has decreased above this. This cooling of the upper layers has brought down the ABL top.

To gain some insight into the TKE, the contribution of the various terms in the TKE budget equation, namely $\overline{w^{\prime} u^{\prime}} \frac{\partial u}{\partial Z}, \overline{w^{\prime} v^{\prime}} \frac{\partial v}{\partial Z}$ and $-(g / \bar{\theta}) \overline{w^{\prime} \theta^{\prime}}$ have been plotted in figures 6 $(a-c)$, in which the variation of the computed sum of the above three terms has also been shown. It is seen that on all the days, the mechanical shear production terms are dominating, particularly the term $\overline{w^{\prime} u^{\prime}} \frac{\partial u}{\partial Z}$, which is mostly a negative term. This suggests that TKE is being produced due to wind shear. The buoyant production term $(g / \bar{\theta}) \overline{w^{\prime} \theta^{\prime}}$ representing the rate of working against buoyancy forces is negative on all the three days indicating that TKE production due to this is suppressed. The sum of these three terms represents the excess of TKE production over consumption. In this case, it should be noted that we have not considered the turbulent transport and pressure correlation terms in the TKE budget equation. These terms are thought to have significant contributions, at least in the surface layer (Wyngaard and Cote 1971). These terms have been included, however, in the second order model, which gives a direct estimate of the TKE. Thus, from figure 6(a), we find that on 19th June, there is a net production of TKE in the shaded zones in the figure, viz. between $50-90 \mathrm{~m}, 110-130 \mathrm{~m}$ and $150-200 \mathrm{~m}$. In between these layers TKE is lost, but as 

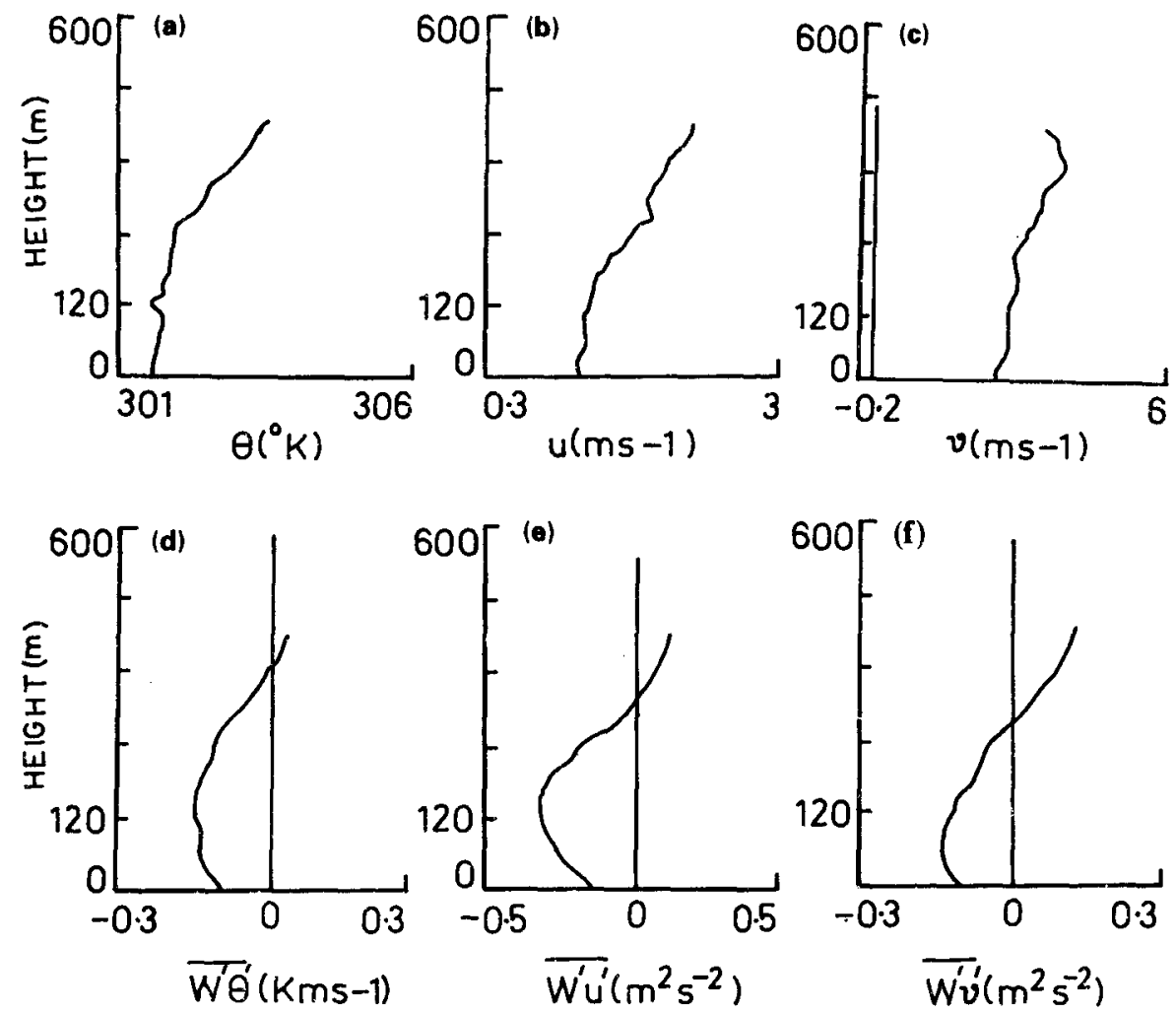

Figure 4. Same as in figure 3 but for 20th June, 1990.

seen from the figure the gain exceeds the loss. The build up of TKE between $110-130 \mathrm{~m}$ and above $160 \mathrm{~m}$ is quite significant on this day. At the lowest levels, gain and loss almost balance each other.

On 20th June, as may be observed from figure 6(b), a large build up of TKE occurs below $60 \mathrm{~m}$, with significant gain between $40-60 \mathrm{~m}$. A considerable amount of TKE seems to be lost above $140 \mathrm{~m}$. On $21 \mathrm{st}$ June, as seen from figure 6(c), where we are dealing with a transition boundary layer, the TKE consumption always exceeds production, with significant losses below $60 \mathrm{~m}$. It may be noted that as defined in the parameterization for turbulent fluxes (equation (2.3)), the potential for mixing is related to the excess of TKE production over consumption and dissipation. So, the shaded zones in figures 6(a) and 6(b) are zones of vigorous turbulent mixing. In contrast, on 21 st June (figure $6 \mathrm{c}$ ) the potential for mixing is quite low, particularly in the lower levels.

At this point, it would be useful to obtain some information regarding the turbulent eddies that are involved in the mixing process. The transport.spectra and the mixing length are two important parameters in this connection. The transport spectra give an idea about the contribution of eddies of various sizes to the flux across any level. The mixing length at any level is a measure of the mean distance moved by the air parcels during the mixing process.

The contribution of the eddies ranging in size between $10-30 \mathrm{~m}, 40-100 \mathrm{~m}, 120$ $170 \mathrm{~m}$ and $180-230 \mathrm{~m}$ to the heat flux across different levels has been illustrated in 

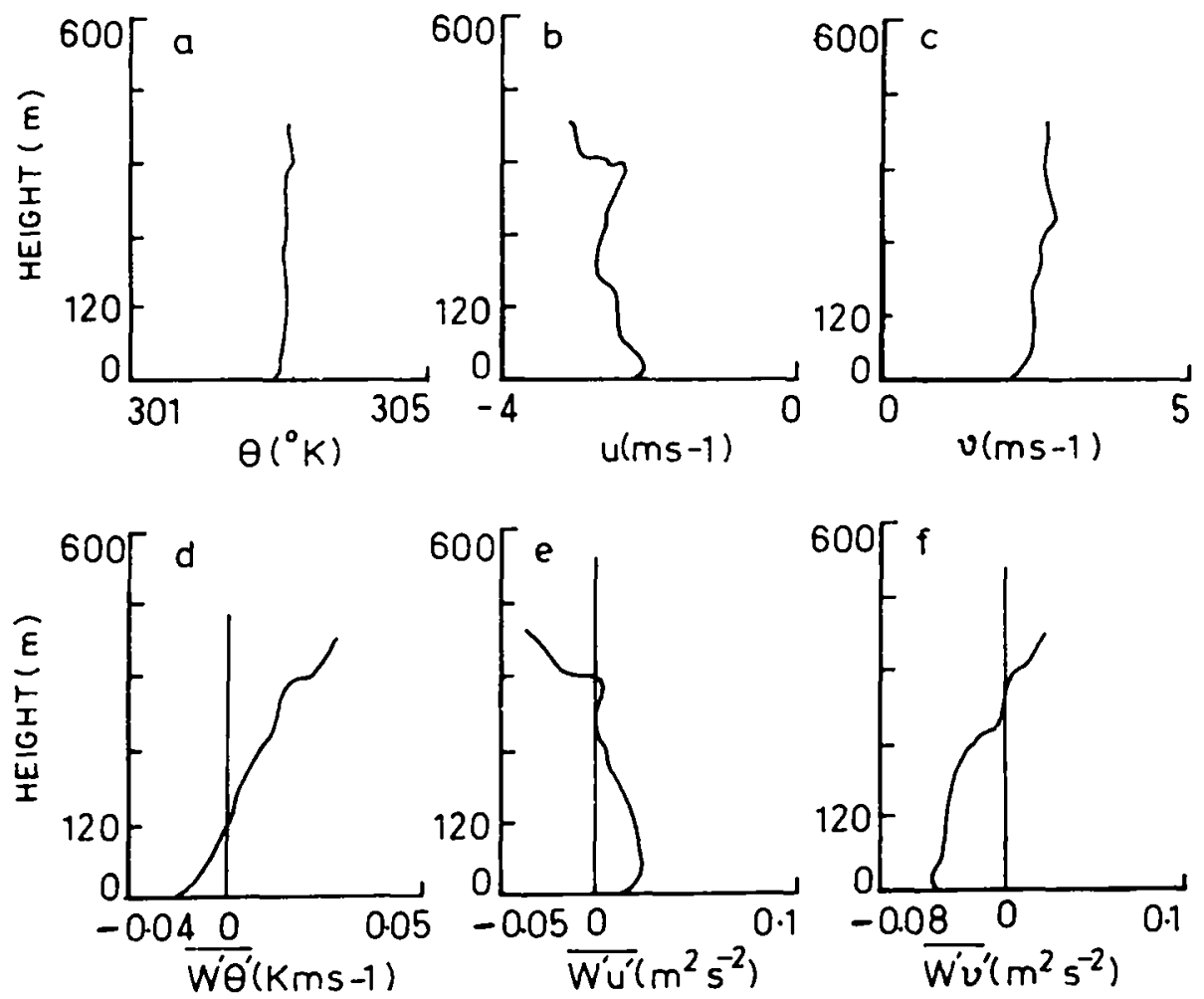

Figure 5. Same as in figure 3 but for 21 st June, 1990.

figures 7(a-b), for 19 th and 20th June respectively, In figure 7(c), the contribution to the heat flux by eddies ranging in sizes between $10-40 \mathrm{~m}, 50-70 \mathrm{~m}$ and $80-100 \mathrm{~m}$ has been shown for 21st June. Recalling that the heat fluxes (figures 3(d), 4(d) and 5(d)) are all negative at least up to the ABL top on all three days, we may conclude that all eddies being considered in figure $7(\mathrm{a}-\mathrm{c})$ are transporting heat downwards.

The major contribution to the downward heat flux throughout the ABL comes from the $120-230 \mathrm{~m}$ eddies for 19 th and 20 th June. The largest contribution up to the level $1 \cdot 1 Z_{i}$ on both days is from the $180-230 \mathrm{~m}$ eddies. At this level, the contribution from the $120-170 \mathrm{~m}$ eddies begins to dominate. The contribution of the $180-230 \mathrm{~m}$ eddies remains nearly constant (but less than that from the $120-170 \mathrm{~m}$ eddies), between $0.9 Z_{i}-0.5 Z_{i}$ on 19 th June (figure $7 \mathrm{a}$ ). On 20 th June, as figure $7(\mathrm{~b})$ indicates, the contribution of these eddies keeps on increasing till $0.8 Z_{i}$, although this contribution is less than that of the $120-170 \mathrm{~m}$ eddies at these levels. Below $0.5 Z_{i}\left(0.8 Z_{i}\right)$ on 19th June (20th June), the contribution to the heat flux by the $180-230 \mathrm{~m}$ class of eddies becomes weaker but increases again below $0.4 Z_{i}\left(0.5 Z_{i}\right)$ where it once more exceeds the contribution from the $120-170 \mathrm{~m}$ class.

The $120-170 \mathrm{~m}$ eddies dominate between $0.9 Z_{i}-0.5 Z_{i}\left(1.04 Z_{i}-0.72 Z_{i}\right)$ on 19 th June (20th June). The smaller eddies belonging to the $40-100 \mathrm{~m}$ class contribute less significantly than the above two classes all throughout the ABL on 19th June. On 20th June however, the contribution of the 40-100 m eddies follows a different pattern, showing increasing contributions at $1.4 Z_{i}$ and again at $0.6 Z_{i}$. Figure 7 (c), for 21 st June, 

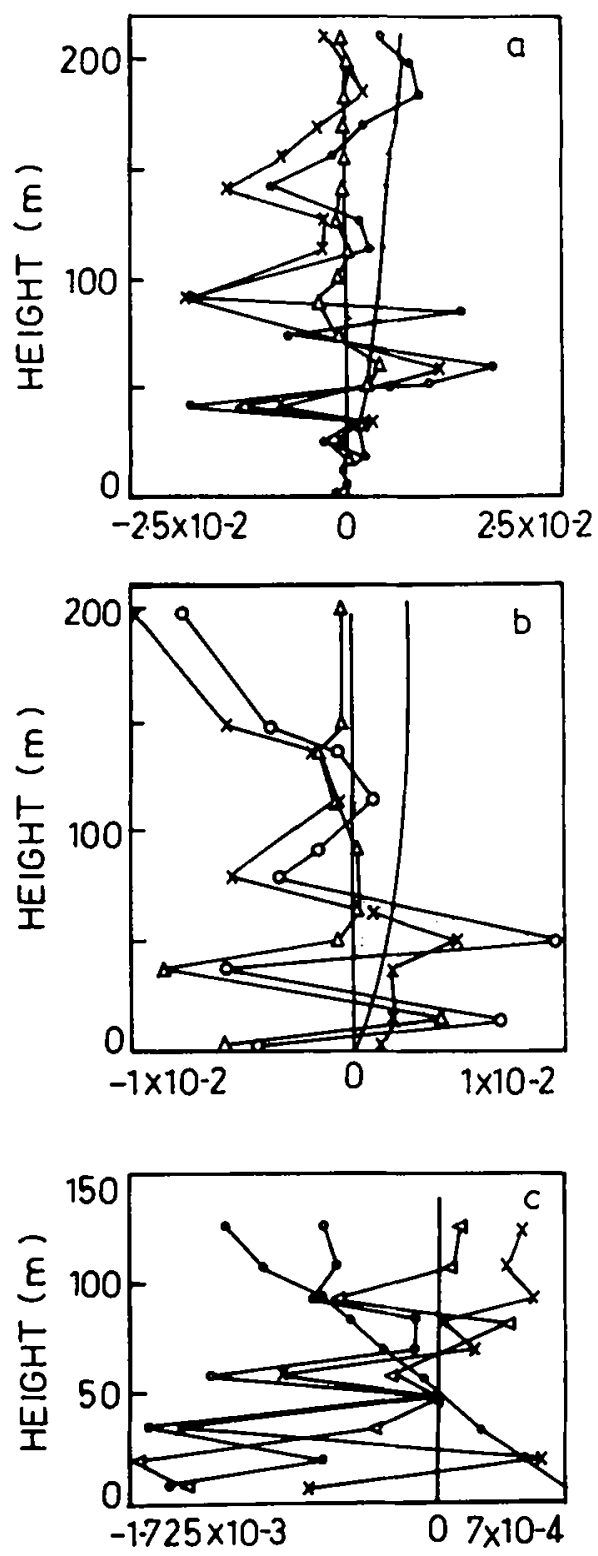

Figure 6. Turbulence kinetic energy budgets for: a) 19th June. b) 20th June and c) 21 st June. buoyant production; $x--\times-x$ mechanical shear production (7onal): $\triangle$. $\triangle-\triangle$ mechanical shear production (meridional): $0 \cdot 0-0$ total of all terms.

reveals much the same pattern. However, on this day, the largest class of eddies, $80 \cdots 100 \mathrm{~m}$, contributed significantly to the heat flux as low as $0 \cdot 2 Z_{i}$. The major contribution (between $0.7 Z_{i}-0.4 Z_{i}$ ) comes from the $50-70 \mathrm{~m}$ eddies on this day. Below this, all the eddies are seen to contribute to the heat flux with the $10-40 \mathrm{~m}$ eddies dominating below $0 \cdot 25 Z_{i}$. Thus only on 21 st June, when we are dealing with a transition boundary layer in the late afternoon, we find that the smallest class of eddies contributes largely in the downward 

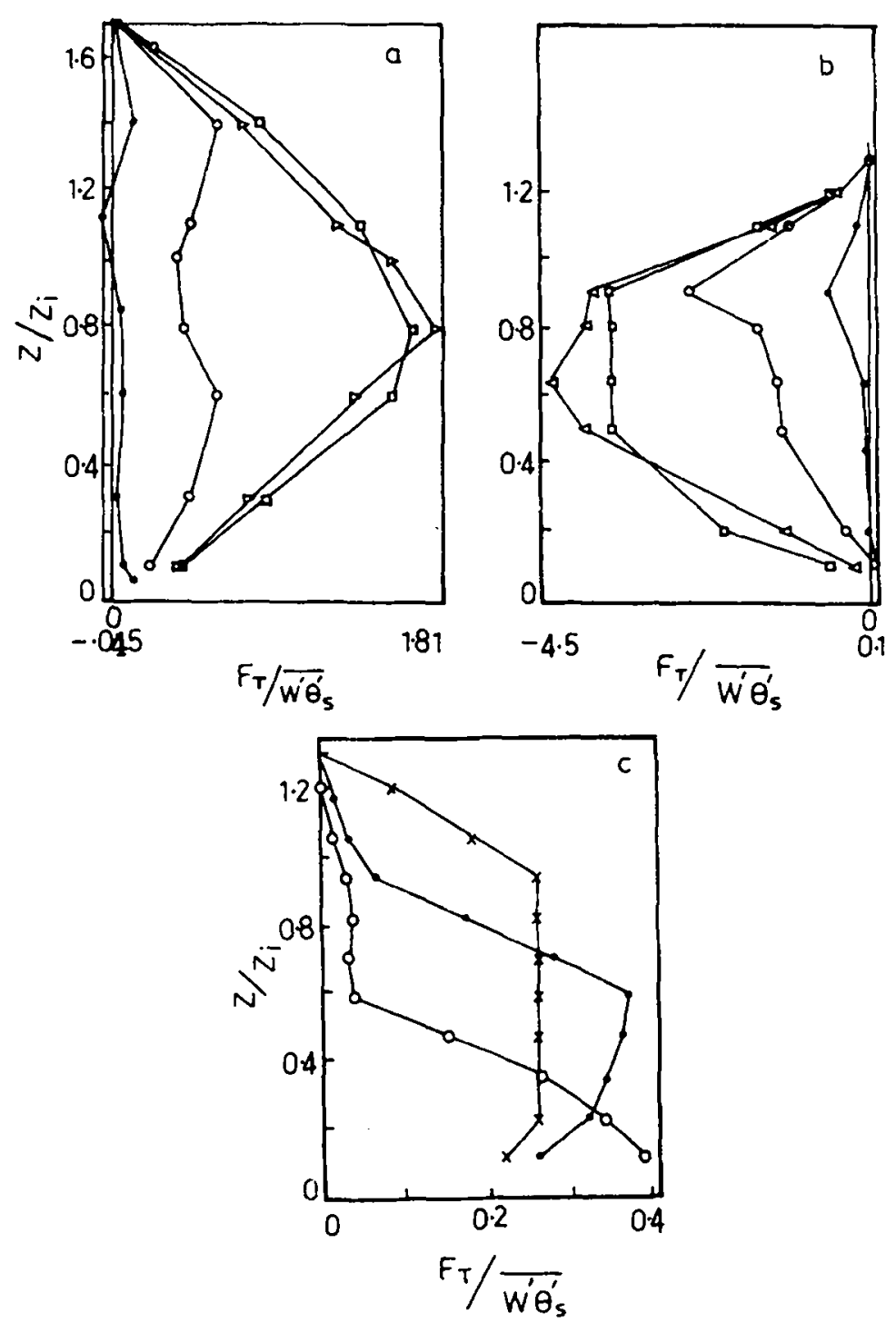

Figure 7. Contribution of turbulent eddies of various sizes to the heat flux across any level: a) 19(h June. b) 20 th June, c) $21 \mathrm{st}$ June. In a) and b): $10.30 \mathrm{~m}, 0-0-040-100 \mathrm{~m}$. $\triangle-\triangle-\triangle 120-170 \mathrm{~m}, \square-\square-\square] 180-230 \mathrm{~m}$. In cl: $\bigcirc-O-O 10-40 \mathrm{~m}$,

s0-70 m. $\times$-... $\times$-.. $\times-80-100 \mathrm{~m}$.

heat transfer in the surface layer, whereas, in the early morning ABL's of 19th and 20th June, all eddy sizes considered, contribute in the surface layer.

We next consider the mixing length profiles for 19 th -20 th June, illustrated in figure 8 . A zone of vigorous mixing exists in the lowest $50 \mathrm{~m}$ of the ABL on both these days. This agrees with the earlier finding that the zone below $60 \mathrm{~m}$ is a zone of TKE production and vigorous mixing (figures $6 \mathrm{a}$ and $\mathrm{b}$ ). Above this level, on 19th June, the vigour of mixing decreases around the $55 \mathrm{~m}$ level, which again corresponds to the zone of TKE consumption shown in figure 6(a). The largest values of the mixing length occur 


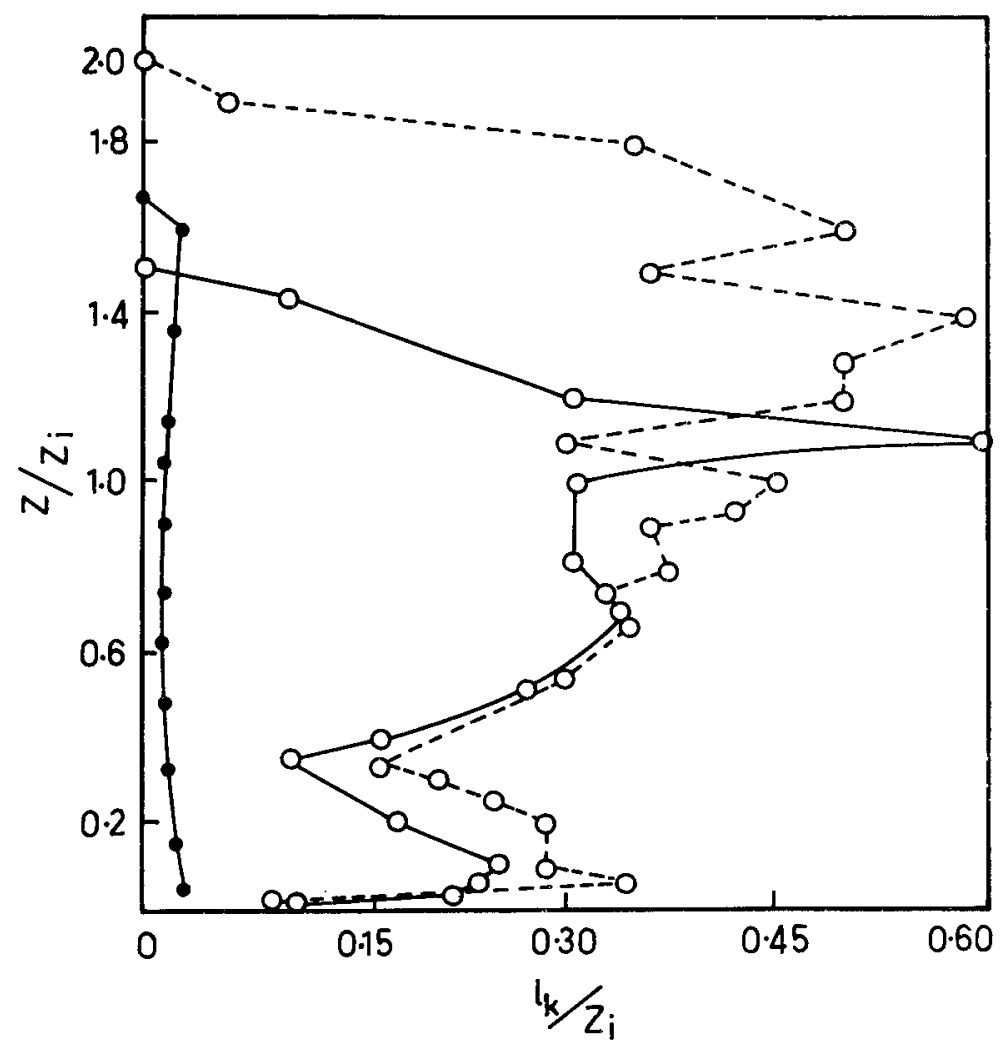

Figure 8. Total mixing length profiles for Kharagpur. $\mathrm{O}-\mathrm{O}-\mathrm{O}$ 19th June, $\mathrm{O}-\mathrm{O}^{--} \mathrm{O}$ 20th June, 21 st. June.

near the ABL tops on both the days. The mixing also appears to be more intense on 20 th June than on 19th June. The mixing length profiles for 21st June, again display a different pattern. The amount of mixing is relatively more below $35 \mathrm{~m}$ although far less than that on 19th, 20th June, and increases again near the ABL top, after which it decreases steadily. In between, mixing is highly suppressed. This can perhaps be attributed to the TKE consumption exceeding production throughout the ABL on this day.

Finally, we have shown the one-dimensional anisotropy of turbulence in figures $9(\mathrm{a}-\mathrm{c})$. We recall that on all three days, the lowest levels in the ABL had a very weak or near-neutral stratification with stable layers above. The transilient matrix obtained in this situation was symmetric, in contrast to the matrices obtained in convective situations. Consequently, partitioning of the mixing length into upward and downward components for each source-destination box, resulted in $l_{j} \uparrow=l_{i} \downarrow$ and $l_{j} \downarrow=l_{i} \uparrow$. Nevertheless, since $l_{j} \uparrow$ is not equal to $l_{j} \downarrow$, the anisotropic structure of the turbulent eddies is highlighted.

Figures 9(a and b) reveal that for the early morning boundary layers on 19th and 20th June, the downward transport dominates the upward transport at all but the lowest levels. The maximum downward transport occurs from the level $0.9 Z_{i}$ on 19 th June, upward transport on that day being confined mostly to below $0.4 Z_{i}$. On 20 th June, downward transport is maximum from the $1.5 Z_{i}$ level and the ABL top. Upward 

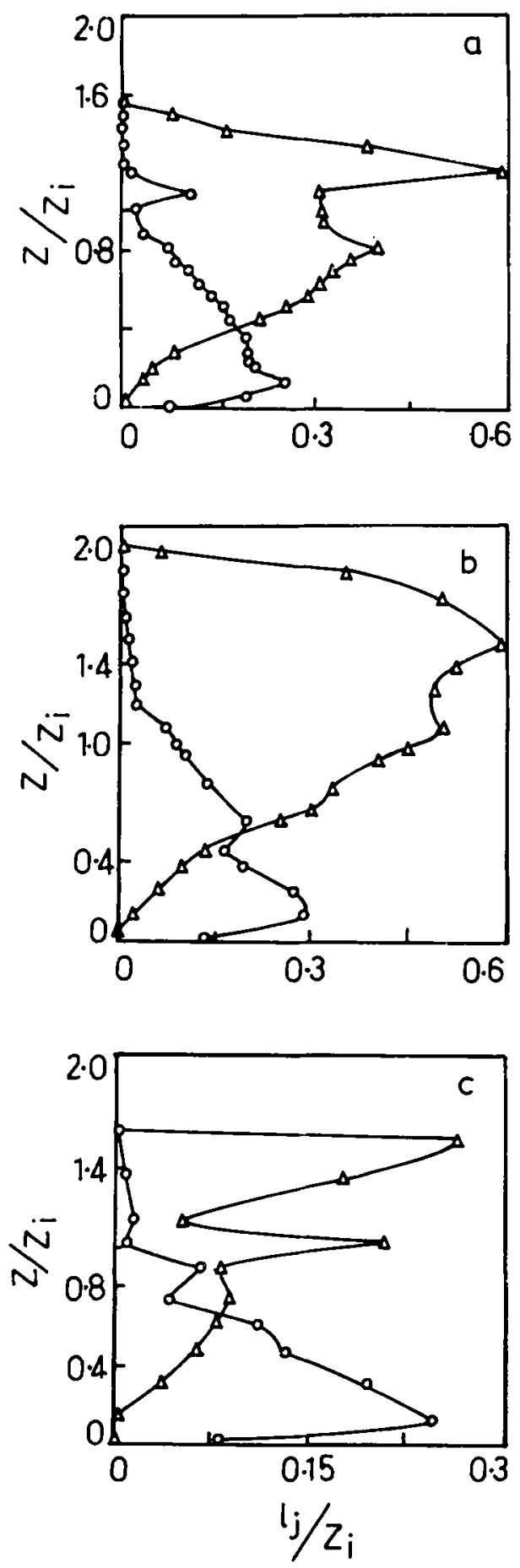

Figure 9. One-dimensional anisotropy of turbulent eddies over Kharagpur: a) 19th June. b) 20 th June and c) 21 st June. $O-O-O l_{j} \uparrow \Delta^{--} \Delta^{--} \Delta l_{j} \downarrow$. 

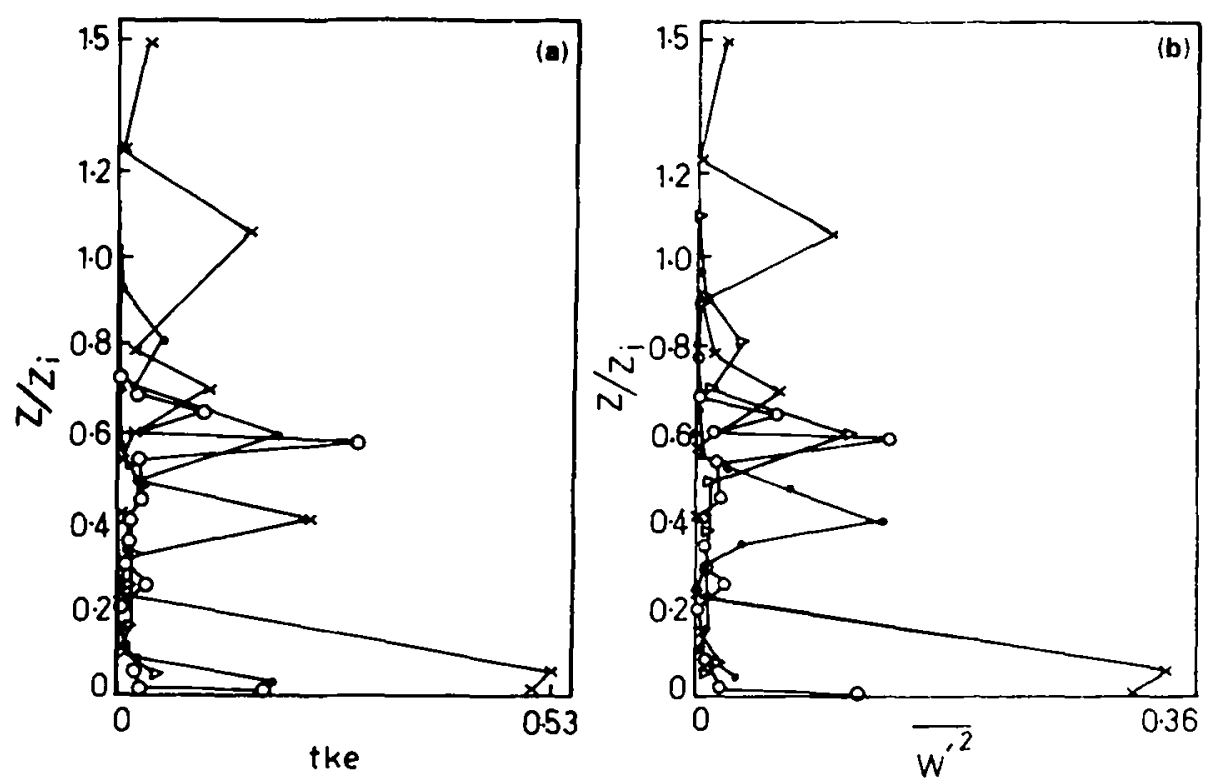

Figure 10. Outputs from the second order model. a) Turbulence kinetic energy and b) Turbulent vertical velocity variance. $O-O^{--} \mathrm{O} 17 \mathrm{~h}$ Junc. 18 th June. $\angle$ $\triangle-\triangle 19$ th June, $x-x-\times 21$ st June.

transport is quite strong for this day and exceeds the downward component below $0.23 Z_{i}$, and equals it around $0.23 Z_{i}-0.27 Z_{i}$. As figure $9(\mathrm{c})$ indicates, for the lati afternoon transition boundary layer on 21 st June, the upward transport dominates up to $0.73 Z_{i}$, above which downward transport dominates between $1.6 Z_{i}$ and the ABL iop.

We now discuss some of the results obtained from the second order closure model. With the turbulence length scale $\Lambda$ kept constant, the profiles of the TKE and turbulence vertical velocity variance $\overline{w^{\prime 2}}$ obtained for $17 \mathrm{th}, 18 \mathrm{th}, 19 \mathrm{th}$ and $21 \mathrm{st}$ June, 1990 have been displayed in figures 10 ( $\mathrm{a}$ and $\mathrm{b}$ ) respectively. The most interesting feature observed here is the one-to-one correspondence of the TKE and $\overline{w^{\prime 2}}$ profiles. In particular, the profiles for $17 \mathrm{th}, 18 \mathrm{th}$ and $21 \mathrm{st}$ June indicate distinct and separate $\%$ nes of TKE and corresponding $\overline{w^{\prime 2}}$ maxima. This indicates the existence of zones of turbulence, in other words, patchy turbulence, a common and well-known feature in stable boundary layers.

For 20 th June, the second numerical experiment mentioned in $\$ 3$ was conducted. Two model runs were made, one (experiment $A$ ) using prescribed inputs of heat and momentum, exactly similar to the ones provided in the transilient model run for that day. In the second experiment (experiment $B$ ) a higher value of heat flux and lower momentum flux were used. The profile of the length scale $\Lambda$ that agrees well with the mixing length profiles from the transilient model has been plotted in figure 11 . Turbulence is seen to be most vigorous at the lower levels, namely $0 \cdot 14 Z_{i}$ and $0.23 Z_{i}$ for the experiments $\mathrm{A}$ and $\mathrm{B}$ respectively. Another turbulent zone occurs around $0.4 Z_{i}$ (experiment $A$ ) and $0.63 Z_{i}$ (experiment $B$ ). Above these levels, turbulence decreases to a minimum at the $A B L$ top and increases above it. Comparison of figure 11 with figures $6(\mathrm{~b})$ and 8 shows that the levels of turbulent activity below $0.5 Z_{i}$ agree fairly 


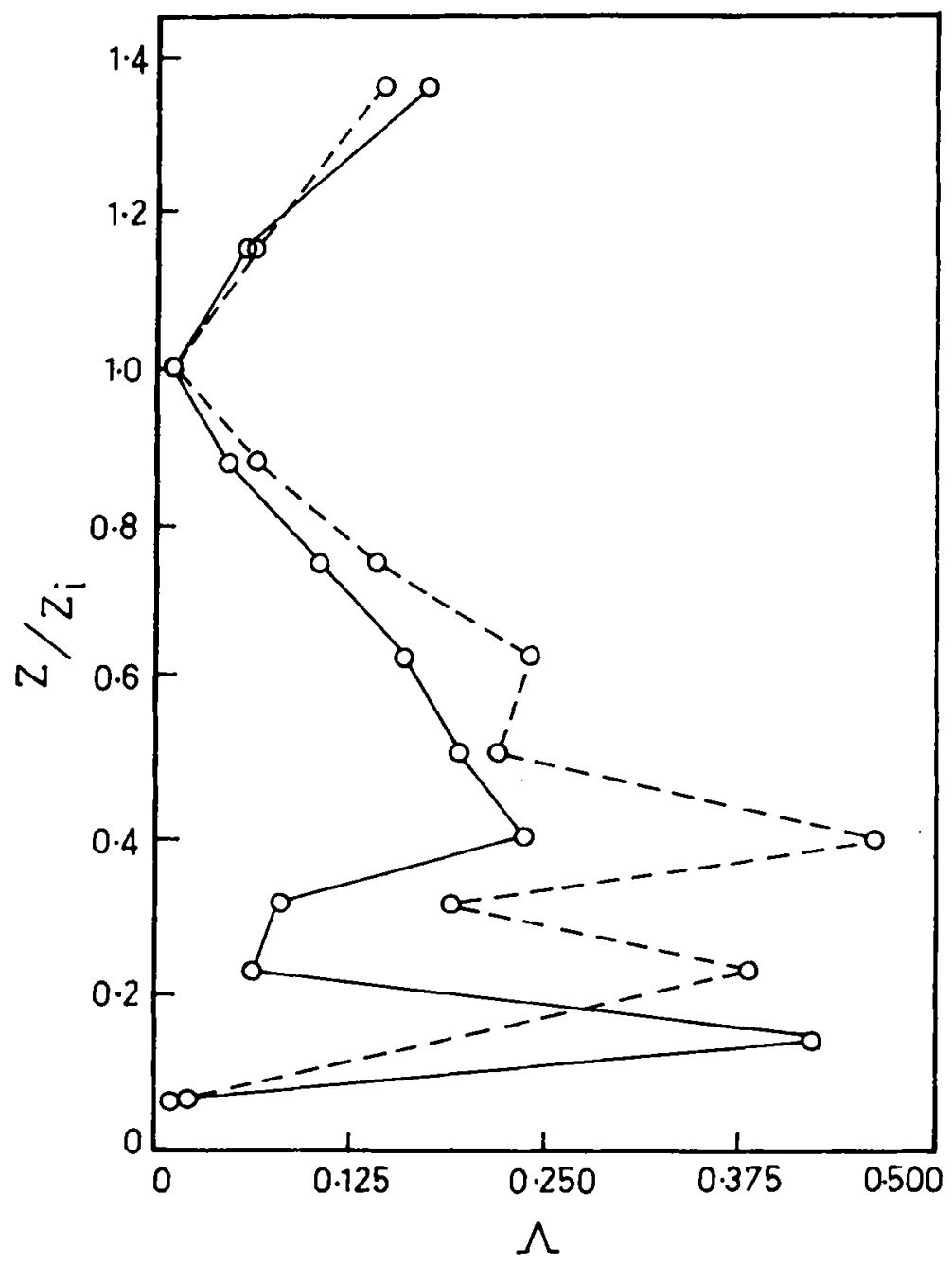

Figure 11. Profiles of the characteristic turbulent length scale $\Lambda$, obtained from the second order model for 20th June.

O $\cdots$ O--O Experiment A: Prescribed heat flux:- $.004 \mathrm{~K} \mathrm{~m} / \mathrm{s}$. drag coefficient $C_{D}=0.0039$.

O- - $---O$ Experiment B: Prescribed heat flux:- $.006 \mathrm{~K} \mathrm{~m} / \mathrm{s}$, drag coefficient $C_{D}=0.0015$.

well. It is also interesting to note that at higher levels, the profile for the two experiments come close to each other whereas at lower levels, their difference is significant.

\section{Conclusion}

The case studies for 17 th -21 st June, 1990 reveal certain interesting features as a result of this diagnostic study on the turbulent transport within the ABL. Using the transilient model, the case studies for the early morning ABL's of 19th - 20th June indicate that distinct zones exist where TKE production exceeds consumption and thus support turbulent activity. In contrast, in the late-afternoon transition boundary layer (21st June), 
turbulence is suppressed due to the TKE consumption exceeding production all throughout the ABL. The contribution to the downward heat flux by several classes of eddies indicates that, for 19th-20th June, eddies of all sizes between $40-230 \mathrm{~m}$ contribute up to and within the surface layer, while for 21 st June, only the smallest eddies dominate within the surface layer. The early morning boundary layers are characterised by downward turbuient transport dominating over upward transport while for the late afternoon case, the reverse is true.

The results from the second order model provide diagnostic evidence of zones of patchy turbulence which is another significant upshot of this study.

\section{Acknowledgement}

The authors gratefully thank the Department of Science and Technology, Government of India for providing financial support. The help and suggestions given by Prof. D K Rakshit of Jadavpur University and Dr. K G Verneker of IITM, Pune, are gratefully acknowledged.

The authors also thank Prof. A Prabhu of IISc, Bangalore, and Dr. S Shivramakrishnan of IITM, Pune, for helpful discussions.

Finally we express our thanks to Prof. R Narasimha of IISc, Bangalore for his helpful criticisms regarding the improvement of the manuscript.

\section{References}

Bhumralkar C M 1976 A survey of parameterization techniques for the planetary boundary layer in .atmospheric circulation models. Report R-1653-AR PA JULY 1976. AR PA Order No. 189 -1. 6P10 Information Processing Technique Office, Rand Corp. Santa Monica, CA 90406, 84 pp.

Deardorff J W 1972 Numerical investigation of neutral and unstable planetary boundary layers. $J$. Atmos. Sci. $2991-115$

Deardorff J W 1974: Three dimensional numerical study of turbulence in an entraining mixed layer. Boundary-Layer. Meteorol. 7 199-226

Donaldson C duP 1973 Construction of a dynamic model of the production of atmospheric turbulence and dispersal of atmospheric pollutants. Workshop on Micrometeorology: (ed) D A Haugen (AMS, Boston) pp. $313-392$

Hogstrom U 1990 Analysis of turbulence structure in the surface layer with a modified similarity formulation for near neutral conditions. J. Atmos. Sci. 47 1949-1972

Keller L V and Friedman A A 1924 Differentialgleichung fur die turbulente Bewegung einer Kompressiblen Flussigkeit. Proc. 1st Int. Cong. Appl. Mech., Delft. pp. 345-405

McBean G A (ed.) 1979 The Planetary Boundary Layer. WMO Technical Note No. 165, 201 pp.

Neiuwstadt F T M and Van Dop H (eds) 1984 Atmospheric Turbulence and Air Pollution Modelling (Dordrecht, Holland: D Reidel Publishing Company) $358 \mathrm{pp}$.

Stull R B 1984 Transilient turbulence theory, Part 1: The concept of eddy mixing across finite distances. $J$. Atmos. Sci. $₫ 1$ 3351-3367

Stull R B and Driedonks A G M 1987 Applications of the transilient turbulence parameterization to atmospheric boundary layer simulations. Boundary-Layer Meteorol. 40 209-239

Stull R B 1988 An Introduction to Boundary Layer Meteorology (Dordrecht, Holland: Kluwer Academic Publishers) pp. 666

Stull R B 1991 Static stability - An update. Bull. Am. Meteorcl. Soc., $721521 \cdot 1529$

Stull R B 1992 Review of transilient turbulence theory and non-local mixing. Boundary-Layer Meteorol. 45

Wyngaard J C and Cote O R 1971 Budgets of turbulent kinetic energy and temperature variance in the atmospheric surface layer. J. Atmos. Sci. 28 190-201

Wyngaard J C and Brost R A 1984 Top-down and bottom-up diffusion of a scalar in the convective boundary layer. J. Atmos. Sci. 41 102-112 